Textile applications of commercial photochromic dyes: part 7. A statistical investigation of the influence of photochromic dyes on the mechanical properties of thermoplastic fibres

Basel Younes, Stephanie C. Ward, Robert M. Christie \& Samantha Vettese 


\title{
Textile applications of commercial photochromic dyes: part 7. A statistical investigation of the influence of photochromic dyes on the mechanical properties of thermoplastic fibres
}

\author{
Basel Younes ${ }^{a}$, Stephanie C. Ward ${ }^{b}$, Robert M. Christie ${ }^{c}$ and Samantha Vettese ${ }^{d}$ \\ Department of Textile Industries Mechanical Engineering and Their Technology, Faculty of Mechanical and Electrical Engineering, \\ Damascus University, Damascus, Syria; bSchool of Textiles and Design, Heriot Watt University, Galashiels, UK; 'Heriot Watt University,

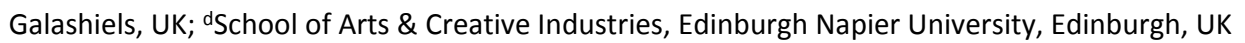

\begin{abstract}
The influence of the concentration of a commercial photochromic dye, incorporated into as-spun and drawn fibres produced from polyethylene and polypropylene, on their mechanical properties was investigated. As-spun fibres were produced by incorporating a commercial photochromic dye at different concentrations at the extrusion stage of the production process of polypropylene, polyethylene and polyester. The fibres from polypropylene and polyethylene, which showed photochromism when exposed to UV light, were then drawn, and their mechanical properties evaluated and analysed using a factorial experimental design, applying appropriate statistical methods. No further investigation was carried out on the polyester as the dye appeared to degrade at the higher temperatures required for its extrusion, leading to a brown fibre, which was not photochromic. According to the analyses, the most significant parameter influencing the mechanical properties of the as-spun fibres was the polymer type; the effect of the dye concentration was dominated by the significance of the polymer type. The effects of the multistage hot-drawing process used to produce the fibres on the mechanical properties of the drawn polypropylene and polyethylene fibres were characterised and statistically modelled. It was found that the dyeing concentration had a negative effect on the tenacity and the modulus of the drawn polypropylene fibres but a positive effect on the tenacity and the modulus of the drawn polyethylene fibres. A dye concentration of $0.2 \%$ on mass of polymer had a positive effect on the elongation of the drawn polypropylene and polyethylene fibres, whereas the higher dye concentration of $0.4 \%$ had a negative effect on the elongation of both drawn fibres. A new forecasting data source was obtained to optimise the selection of the polymer and the dye concentration and to specify the direction of modification, either increasing or decreasing, the significant process parameters.
\end{abstract}




\section{Introduction}

Chromic materials are dyes or pigments that exhibit colour change when exposed to an external stimulus, especially when the change is reversible and controllable. Photochromic dyes undergo colour change when activated by UV irradiation. In the most important industrial dyes, the change is from colourless to coloured, and they revert thermally to their original colourless state when the UV source is removed. Photochromism is of interest because of its commercial importance in responsive eyewear, and its potential for applications in security printing, optical data storage, optical switching devices, cosmetics, solar energy storage and biological systems (Berkovic, Krongauz, \& Weiss, 2000; Christie et al., 2005; Corns, Partington, \& DTowns, 2009; Crano \& Gugliemetti, 1999). Recent interest in photochromic textiles is associated with their potential for application in creative and intelligent textile design, and in functional or smart textile products, for example in brand protection, responsive camouflage and as UV sensors (Aldib \& Christie, 2011, 2013; Billah, Christie, \& Morgan, 2008; Billah, Christie, \& Shamey, 2008; Christie, 2013; Lee, Son, Suh, Lee, \& Kim, 2006; Little \& Christie, 2010, 2011, 2016; Son, Park, Park, Shin, \& Kim, 2007; Wang \& Wu, 1997). The fact that this interest has not led to significant commercial development is arguably due to technical issues associated with application methodology and performance (Corns et al., 2009). This series of publications is aimed, therefore, at establishing how commercial photochromic dyes are capable of performing in textiles following judicious optimisation of formulations and application conditions. In the first three papers, we reported our study of commercial dyes applied by screen printing to textiles using a pigment printing formulation (Billah, Christie, \& Morgan, 2008; Billah, Christie, \& Shamey, 2008; Little \& Christie, 2010), including the development of a colour measurement technology for objective assessment of the performance of photochromic textiles. Two subsequent papers dealt with their application as disperse dyes in the coloration of polyester fabric (Little \& Christie, 2010, 2011). In the most recent paper, we extended the study to the incorporation of two commercial dyes by extrusion into polypropylene to form photochromic fibres, and an assessment of their photochromic performance in the as-spun fibres (Little \& Christie, 2016). Photochromic polypropylene has been described previously in a few patents, but there is little reported in open literature, especially using commercial dyes (Nechwatal \&

Nicolai, 2011).

The technology of synthetic fibres has advanced significantly in recent decades, opening up a wide range of engineering applications. In particular, the development of fibres that incorporate smart technology has become an important goal of modern textile industries. The synthetic fibres, polypropylene, polyethylene and polyester, notably poly(ethylene terephthalate), are used in a wide range of applications, due to their good technical performance and relatively low cost of production, which involves melt extrusion (Mather \& Wardman, 2011). As the molten polymer emerges from the high temperature extrusion process, it is cooled by quenching, either by air or in water. The structure of the as-spun synthetic fibres obtained, including important physicochemical features such as molecular orientation and degree of crystallinity, is developed during the melt-spinning processes. The as-spun fibres produced are required to have a structure that can be drawn easily. Drawing, which may be applied either directly after the extrusion operation or during texturing, is a vital stage in the manufacture of synthetic fibres because it stabilises the structure and strengthens the yarn by improving the molecular orientation and crystallinity (Lord, 2003), as the polymer chains move in the direction of drawing to become oriented parallel to the fibre axis. As the temperature is lowered in the relaxation stage, the polymer chains lose their freedom of movement and thus become fixed. Melt-spinning systems

usually incorporate two or more drawing zones. The drawing ratio that is appropriate in a particular case depends on the drawing conditions and original structure of the filaments (Hes \& Ursiny, 1994) .

Measurement, feedback and adjustment, prediction and correction are main elements in online quality control (Tanguchi, 1986), aiming to satisfy customers requirements and expectations. The use of practical, software-based approaches has improved confidence levels in experimental design and simulation. It is also useful to apply these techniques in manufacturing processes for new products, assisting the engineering by reducing process factor variation (Vlachogiannis \& Roy, 2005). Statistical experimental design (SED) was first developed by Fisher in his agriculture research in the UK in the 1920s. SED analysis offers advantages in addressing the problems arising in traditional analytical approaches that depend on a 'one factor-at-a-time' method. In SED, process factors may be classified as controllable, noise and constant. The noise factors may be classified as, firstly, external (environmental) factors, such as temperature, humidity, dust, supply voltage, electromagnetic interaction, vibrations of instrument supports, sunlight intensity through windows and human error, secondly, unitto-unit variations such as resistance, and finally deterioration in the product as time passes (Phadke, 1989). The focus of SED is on the optimisation of the 
average response values that depend on the factors and their levels. Design and analysis of experimental methods have been shown to provide additional advantages in the analysis of manufacturing processes, such as in the fibreto-fabric engineering approach (El-Mogahzy, 2009). The use of experimental design concepts remains largely limited to large industrial scale applications, but merits wider attention from improved acceptance and understanding of the potential value of the methods, and collaboration between academic and industrial organisations (Gardiner \& Gettinby, 1998).

Unlike polyester, conventional polypropylene and polyethylene fibres cannot be dyed by the traditional dyeing methods used for textiles. However, dyes or pigments may be incorporated by addition at the fibre extrusion stage, a process referred to as mass coloration. We have recently reported an investigation of the mass coloration of polypropylene using photochromic dyes. In that study, two commercially available photochromic dyes were successfully incorporated into polypropylene fibres by melt extrusion to produce fibres that showed striking, intense photochromism. The performance of the fibres, especially in terms of photocolorability and photostability, was evaluated to provide data that are useful in assessing the suitability of photochromic dyes for applications in polypropylene fibres, and to develop approaches to optimisation of performance for specific end-uses. As an extension of this investigation, we now report on a study of the effect of the incorporation of the dyes on the mechanical performance of the fibres, which was analysed on the basis of a SED approach.

\section{Experimental}

\subsection{Materials}

The investigation involved the thermoplastic polymers, polypropylene, polyethylene and polyester. The polymers were supplied in the form of spherical granules, with diameters in the range 3-5 mm. Polypropylene, with MFI of $22.6 \mathrm{~g} / 10$ min., was supplied by Lyondell Basel Industrial Holdings B.V. The polymer is an isotactic polypropylene, designed for extrusion applications for the production of continuous filaments, bulk continuous filaments and fine denier staple fibres. The polymer has a narrow molecular weight distribution and is formulated with an anti-gas-fading stabilisation package. Low-density polyethylene, with MFI 13 g/10 min, was supplied by Exxon Mobil Company. A commercial sample of polyester, poly(ethylene terephthalate) polymer was used; detail concerning its composition was not available from the supplier. A blue commercial photochromic dye belonging to the spiro[2,1-b]oxazine chemical type was used; its trade name is not disclosed for commercial reasons.

\subsection{Extrusion of as-spun fibres by melt spinning}

Fibres were extruded by melt-spinning using a lab-spin machine provided by Extrusion Systems Limited, UK. A specially designed spinneret of 37 holes $\left(0.4 \mathrm{~mm}\right.$ diameter, $\left.\mathrm{l} / \mathrm{d}^{1} 1 / 41.6\right)$ was used. The six heating zones in the extrusion machine profile were set at 190, 200, 210, 220, 230 and $230 \mathrm{C}$ for polypropylene, 180, 200, 210, 220, 230, and 230 C for polyethylene, and 200, 220, 240, 260, 260 and $260 \mathrm{C}$ for polyester. Prior to extrusion, the dye powder, at the appropriate concentration, and polymer pellets were mixed intimately by tumbling in a glass jar on rollers for 30 min to ensure a uniform distribution. The molten polymer, with or without the dye incorporated, was forced through the spinneret as fine jets with the speed, adjusted by the metering pump, fixed at $12 \mathrm{rpm}$ at a pre-pump pressure of 1000 psi. The air cooling quench speed percentage was set at $50 \%$. Spin finish oil, Vickers 1031 , was used in the spinning and drawing processes. The spin finish was diluted fivefold with water before use. Godets and the winders were set at a speed of $100 \mathrm{~m} / \mathrm{min}$, and were controlled independently by DC motors.

\subsection{Multi-stage hot drawing of as-spun fibres}

For technical experimental reasons, the spinning and drawing processes were operated separately. After the as-spun fibres were extruded, hot drawing was carried out on an ESL multi-stage draw frame, supplied by Extrusion System Ltd. The multi-stage draw frame (Figure 1) consists of four hot rollers (160 mm), three hot plates $(49530 \mathrm{~mm})$ mounted between the rollers, a spin finish applicator and a winder. Drawing was carried out at temperatures above the glass transition temperature, $\mathrm{T}_{\mathrm{g}}$, of the polymer. Theoretically, there is a solid-state (actual) draw ratio $\mathrm{R}$, and an adjusted (machine) drawing ratio $R_{D}$ as given by:

$$
\mathrm{R}^{1 / 4 \mathrm{~L} L^{2}}{ }_{\mathrm{L} 1}^{2} ; \mathrm{R}_{\mathrm{D}}{ }^{1 / 4} \mathrm{~V}^{\mathrm{V}} \mathrm{V}_{1} \text { where } \mathrm{V}_{1}{ }^{1 / 4} \text { speed of the first drawing roll (m/min), } \mathrm{V}_{2}{ }^{1 / 4}
$$

speed of the second drawing roll $(\mathrm{m} / \mathrm{min}), L_{1} 1 / 4$ fibre length before drawing, $L_{2} 1 / 4$ fibre length after drawing. 
In the drawing process, the same drawing temperature was applied for both the first and the second stages. This feature assisted in decreasing the number of interactions between factors in the SED process.

\subsubsection{Mechanical properties of fibres}

Tensile testing of the drawn fibres was carried out using an Instron tester (model 3345), equipped with Instron Bluehill V 2.21 software, at a temperature of $20 \pm 2 \mathrm{C}$ and relative humidity $65 \pm 5 \%$ in a conditioned laboratory. The samples were conditioned for $48 \mathrm{~h}$ before testing. An initial gauge length of $20 \mathrm{~mm}$ was stretched at a constant cross head speed of $120 \mathrm{~mm} / \mathrm{min}$. Pre-tension of $0.005 \mathrm{~N} / \mathrm{tex}$ was applied to the yarn to provide a reproducible extension value. After securing the specimen in the grips, the cardboard frame used was cut along the cutting line and load was applied to the fibre. The elongation at break was measured as a percentage of the original length. Samples were taken from different parts of the packages for evaluation

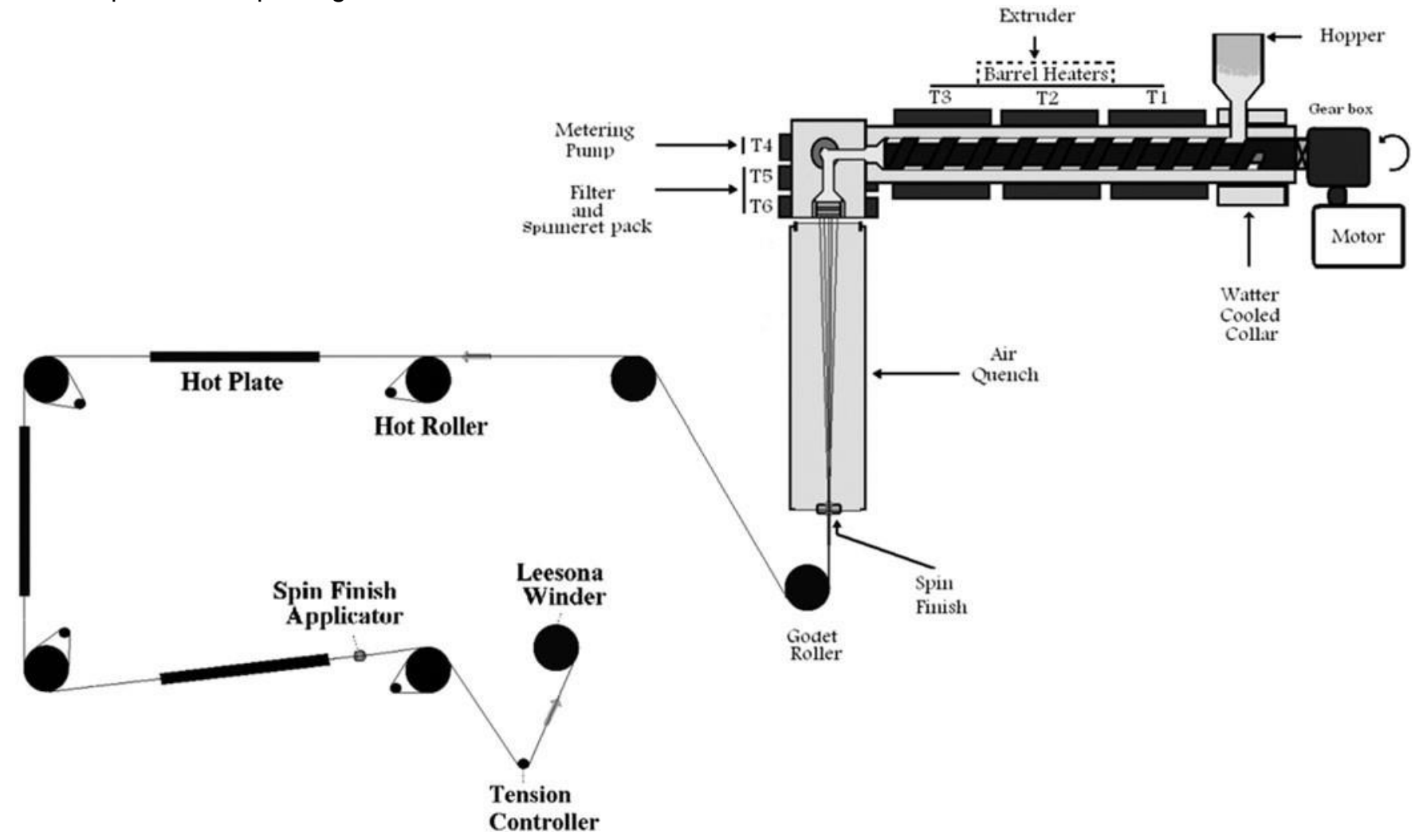

Figure 1. Schematic diagram of the extrusion machine and the multi-stage drawing frame.<smiles>CN1C(=CN=C2C(=O)C=Cc3ccccc32)C(C)(C)C(C)(C)c2ccccc21</smiles> 
Figure 2. The photochromism of spirooxazine (I).

Table 1. Control factors and the levels used in the experimental design.

\begin{tabular}{lcccc}
\hline & & & \multicolumn{2}{c}{ Level } \\
\cline { 3 - 5 } Factor abbreviation & Factor & Low & Middle & High \\
\hline A & Polymer & 1: Polypropylene & 2: Polyethylene & $3:$ \\
B & Dye concentration (\% o.m.f) & 0.0 & 0.2 & Polyester \\
\hline
\end{tabular}

and the results were expressed as the average values from five repeated measurements.

When fibres are tested until they break, they pass through several phases, for example the elastic region, yielding, strain hardening, necking and failure. However, the extent of each region in stress-strain space is materialdependent and not all materials exhibit all of the regions (Brody, 1994).

\section{Results and Discussion}

\subsection{Factorial experimental design and results}

The fibres, dyed and undyed, were spun and hot drawn as described in section 2 . They were then characterised in terms of their mechanical properties, and analysis carried out using a fractional factorial design as a function of the process parameters, using appropriate statistical methods to model the effect of processing (Lochner \& Mater, 1990; Phadke, 1989). All statistical-based plots in this study were constructed directly from the raw data using the software programs Statgraphics Plus, version 5.1 and Minitab 15.1.

The dye used belongs to the spironaphtho[2,1b]oxazine type. A generalised structure (I) of this dye class is shown in Figure 2. The scheme also presents an outline mechanism of the photoinitiated conversion to its coloured ring-opened form, illustrated as structure la although other geometric isomers may also be present. It was observed that the polypropylene and polyethylene containing the dye showed a background pale blue colour before UV exposure. This is probably due to thermochromism, resulting in the formation of the coloured form (la) of the photochromic dye at the high application temperature. The evidence for this is that the molten dyed polymer acquired an intense blue colour, which reduced in intensity as it cooled. It is proposed that some dye molecules become fixed in the coloured form within the crystalline structure of the polymer below its glass transition temperature, $T_{g}$, giving rise to a permanent colour. In contrast, the extruded polyester yarns containing the dye acquired a strong brown colour. UV exposure of the polypropylene and polyethylene yarns containing the photochromic dye resulted in a change in the colour of the fibres to a more intense blue.
However, the dyed polyester yarns did not show visible photocoloration under UV exposure, probably due to degradation of the dye at the higher temperatures required for extruding polyester compared with those used for polypropylene and polyethylene.

The two control parameters selected for the experiments were the polymer type and the concentration of the photochromic dye added to the polymer (Table 1). The three levels of the dye concentration, 0.0 (i.e. undyed), 0.2 and $0.4 \%$ o.m.f., were selected based on the outcomes of our previous study carried out on photochromic polypropylene [16].

A full factorial design, with random ordering, was used for the nine screening trials, contained in an L9 design matrix: $3^{2}=9$. The individual trials carried out in this set of experiments, as detailed in Table 2, involved the two selected control parameters and the three concentration parameter levels, and were designed using Statgraphics software. The total drawing ratio (DR3 $1 / 4$ DR 1 DR2 $1 / 4$ V2/ 
Table 2. L9 experimental design array and processes applied for the experimentation.

\begin{tabular}{|c|c|c|c|c|c|}
\hline Trial number & Polymer & Dye concentration (\% o.m.f.) & Applied pr & cesses & \\
\hline 1 & Polyester & 0.4 & Melt-spinning & & \\
\hline 2 & Polypropylene & 0.2 & $\begin{array}{l}\text { Melt-spinning } \\
\text { drawing }\end{array}$ & and & hot \\
\hline 3 & Polypropylene & 0.4 & $\begin{array}{l}\text { Melt-spinning } \\
\text { drawing }\end{array}$ & and & hot \\
\hline 4 & Polyester & 0.2 & Melt-spinning & & \\
\hline 5 & Polyethylene & 0.4 & $\begin{array}{l}\text { Melt-spinning } \\
\text { drawing }\end{array}$ & and & hot \\
\hline 6 & Polyethylene & 0.0 & $\begin{array}{l}\text { Melt-spinning } \\
\text { drawing }\end{array}$ & and & hot \\
\hline 7 & Polyester & 0.0 & Melt-spinning & & \\
\hline 8 & Polypropylene & 0.0 & $\begin{array}{l}\text { Melt-spinning } \\
\text { drawing }\end{array}$ & and & hot \\
\hline 9 & Polyethylene & 0.2 & $\begin{array}{l}\text { Melt-spinning } \\
\text { drawing }\end{array}$ & and & hot \\
\hline
\end{tabular}

V1 V3/V2 $1 / 4 \mathrm{~V} 3 / \mathrm{V} 1$ ) used in the process is given by the ratio of the speed of the second roller (V3 $1 / 4150 \mathrm{~m} / \mathrm{min}$ ) in the second drawing stage to the speed of the first roller of the first drawing stage (V1 $1 / 450 \mathrm{~m} / \mathrm{min})$. The speed of the second roller in the first stage was the same as the speed of the first roller in the second stage (V2). The draw ratio in the first stage (DR1 $1 / 4 \mathrm{~V} 2 / \mathrm{V} 1)$ is larger than that in the second stage (DR2 1/4 V3/V2) (DR1/DR2 2/1)

(Younes, 2012).

The mechanical properties of the fibres produced were measured, the values obtained as an average of five measurements on each sample, which is represented by the random order number given in the first column in the design matrix, as shown in Table 2. The test results are given in Table 3. There are potential errors related to process setting-based variation, tension or slippage on the drawing tension during the preparation of the sample for testing, changes in independent factors, or random causes due to uncontrolled variation. However, the mean values of the measured data obtained from the specimens were found to have acceptable standard deviations.

The tools used for the statistical analysis included Pareto charts, effects and interaction plots, Daniel's plots, and analysis of variance (ANOVA). All statistical analysis and the plots were constructed directly from the raw data using Statgraphics and Minitab software. On the basis of the three-level experiments, the factor and interaction effects were determined from the differences between the average responses at the low, the middle and high levels, after the fibres were drawn.

\subsection{Statistical analysis of the effects of the factors and} their interactions on the responses

Pareto charts are used to illustrate the significance of the factors and their interactions, arranged in decreasing order (y-axis) depending on the significance of the effect
( $x$-axis). Factors can have either a positive ( $p$ ) or negative $(-)$ effect as represented by black and white, respectively. The order of the significance of the factors is presented for each response, with further detail described in the statistical analysis that follows, showing the importance and the direction of the effect, and the way that the factors interact with each other. The vertical line indicates which effects are significant. In the Pareto charts shown in Figure 3, for (a) tenacity, (b) elongation at break and (c) modulus the significance of the factors and their interactions of the drawn fibres are arranged in decreasing order. In terms of the mechanical

Table 3. Results from the testing of the mechanical properties of the fibres.

\begin{tabular}{|c|c|c|c|}
\hline \multirow{2}{*}{$\begin{array}{l}\text { Trial number } \\
1\end{array}$} & \multirow{2}{*}{$\begin{array}{r}\begin{array}{r}\text { Tenacity } \\
\text { (cN/Tex) }\end{array} \\
2.89\end{array}$} & \multicolumn{2}{|c|}{$\begin{array}{r}\text { Elongation at break (\%)Modulus } \\
(\mathrm{cN} / \mathrm{Tex})\end{array}$} \\
\hline & & 1.9 & 19.6 \\
\hline 2 & 3.77 & 439.2 & 1.0 \\
\hline 3 & 3.49 & 445.0 & 1.0 \\
\hline 4 & 2.39 & 1.9 & 11.6 \\
\hline 5 & 2.46 & 155.8 & 5.0 \\
\hline 6 & 2.36 & 148.3 & 3.0 \\
\hline 7 & 3.03 & 1.8 & 18.9 \\
\hline 8 & 3.92 & 420.0 & 1.0 \\
\hline 9 & 2.40 & 150.0 & 5.0 \\
\hline
\end{tabular}

properties, the Pareto charts show that polymer type is the more important factor, affecting the tenacity, elongation and modulus. In addition, the Pareto chart for elongation (Figure 3(b)) shows that the dye concentration has a moderately significant effect on the elongation.

Main effects plots and interaction plots obtained from the statistical analysis are techniques that also provide information on the effects of factors on the responses. The effect line determines the effect of the factors on the basis of the length and the slope of the line between two levels. The longer the line the more significant the effect of the factor. The direction of the effect is determined by the slope of the line. The effect of the factors on the mechanical properties, shown in Figure 4, (a) tenacity, (b) elongation at break and (c) modulus, was obtained from the average responses of the low, middle and high levels, using the design matrix. For example, Figure 4(a) shows 
that the tenacity increases with the use of polypropylene (polymer 1) or by decreasing the dye percentage.

All the interactions were simulated, and plotted to show the existence, or otherwise, of each two factor interaction, as coded in Table 1. To determine the form of the interaction between each two factors and their level, together with how the direction of change of the interacting factors influences the change in the fibre properties, an interaction plot is used, as shown in Figure 5 . The interaction between the polymer type and the dye percentage for tenacity is illustrated in the plot shown in Figure 5(a). The polymer type is presented on the $x$-axis as 1 for polypropylene, 2 for polyethylene and 3 for polyester, while the dye concentration is shown as two different lines, one for the low level $(0.0 \%)$ and the other for the high level $(0.4 \%)$. When the polypropylene is paired with the fibre containing no $(0 \%)$ dye, the maximum tenacity is obtained. The space between the two lines confirms the presence of an interaction. The main effects plot and interaction plot for tenacity, Figures 4(a) and 5(a), respectively, and for elongation, Figures $4(b)$ and $5(b)$, respectively, show that in both cases the polymer type

(a) Standardized Pareto Chart for Tenacity

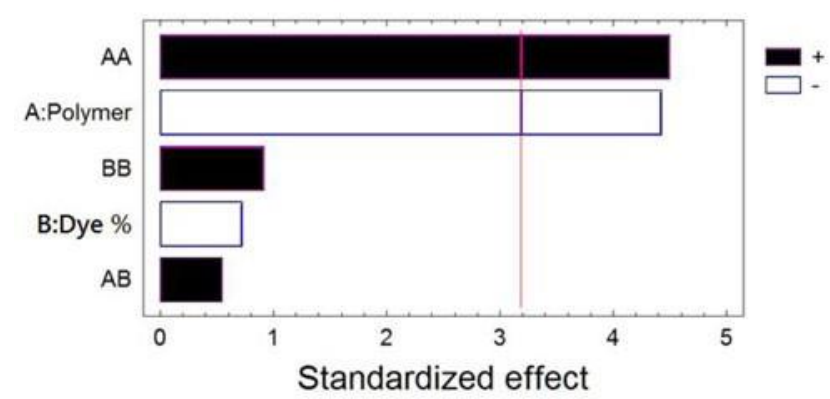

(b) Standardized Pareto Chart for Elongation

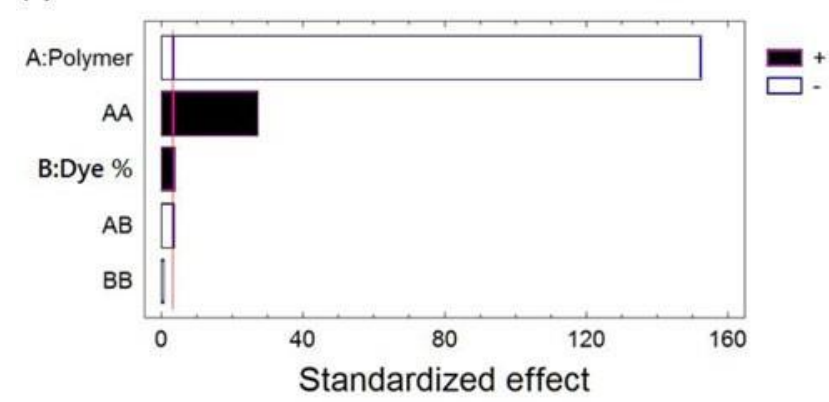

(c) Standardized Pareto Chart for Modulus

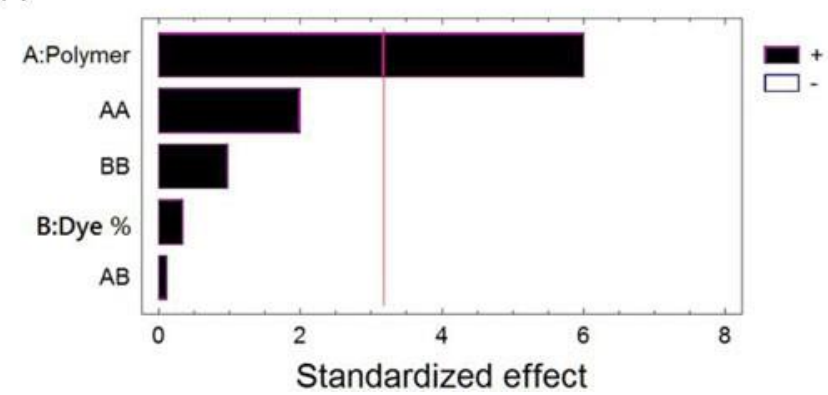

Figure 3. Pareto chart for the tenacity (a), elongation at break (b) and modulus (c). is the more important factor. The same observation is illustrated from the main effects plot and interaction plot for modulus, Figures 4(c) and 5(c), respectively, but in addition there is a noticeable effect from the dye percentage.

The Statgraphics program was also used to calculate statistical standardised percentage order factors and their interaction values, which were then plotted on $x$ - and $y$ axes, respectively, to generate a normal probability plot, also referred to as a Daniel's plot (Figure 6). This technique assists in the separation of factors into either important or unimportant categories and gives further detail on whether the effect of the factors is positive or negative (Gardiner \& Gettinby, 1998). The straight line represents the empirical relationship in the middle of the range. The significance of the effect, either positive or negative, is reflected in the deviation of the data points from the straight line. The further the deviation, the greater the statistical significance. If responses follow a normal distribution pattern, then this means that there are no statistically significant factor effects obtained from the set of experiments. Figure 6 displays the normal probability plot of the estimated responses and illustrates detail concerning the normal distribution of the data. 
(a)

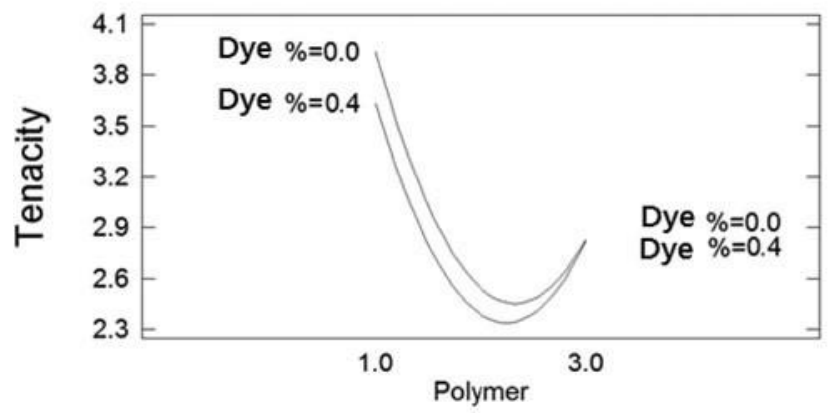

(b)

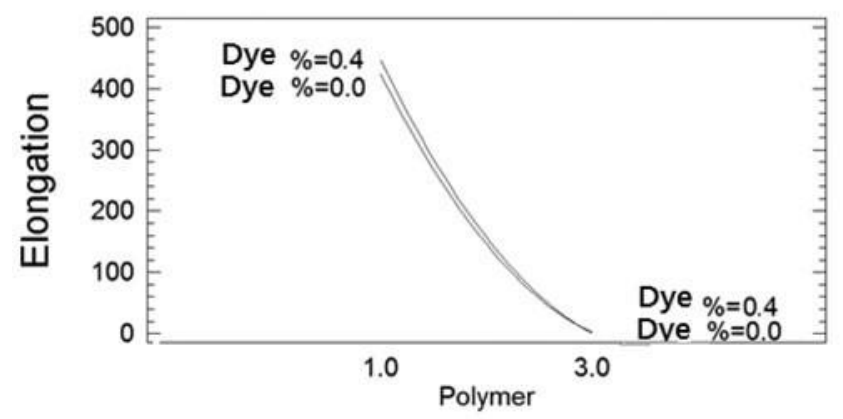

(c)

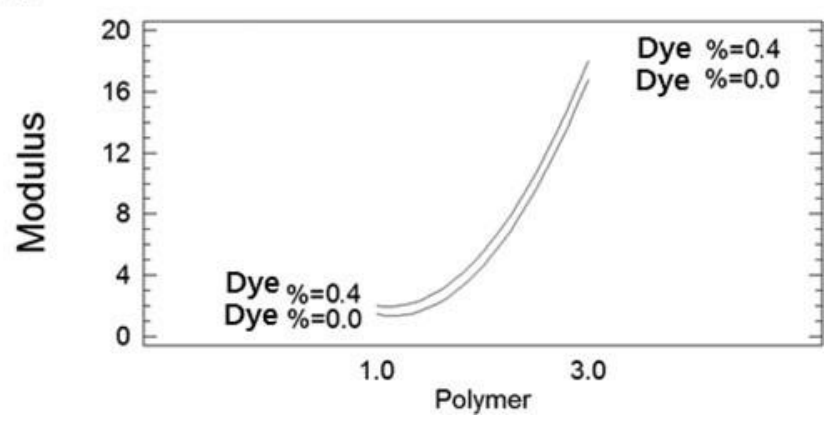

Figure 4. Main effect plots for the tenacity (a), elongation at break (b) and modulus (c).

In terms of mechanical properties, the polymer type has a negative effect on elongation at break and tenacity, and a positive effect on the modulus. Dye concentration has a less important effect on the mechanical properties.

\subsection{Analysis of variance}

To determine the effects of the factors in terms of statistical significance, ANOVA of the data obtained was applied. The ANOVA approach is a mathematical method based on statistical data by which the response data can be compared to the error data and the significance of the independent factors or from their interaction can be assessed. The $p$ value is determined using the graphical method ( $p$ : a-significance level) and can be obtained from statistical analysis (Lochner \& Mater, 1990). A probability or $p$ value used in ANOVA provides quantitative and objective criteria for judging the statistical significance of the effects. Each factor that has a $p$ value less than 0.05 indicates that the factor is significantly different from zero at the $95.0 \%$ confidence level. ANOVA

(a) Interaction Plot for Tenacity

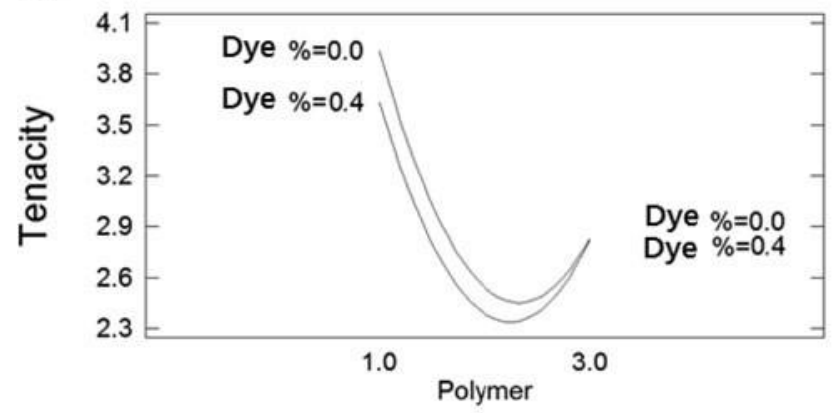

(b)

Interaction Plot for Elongation

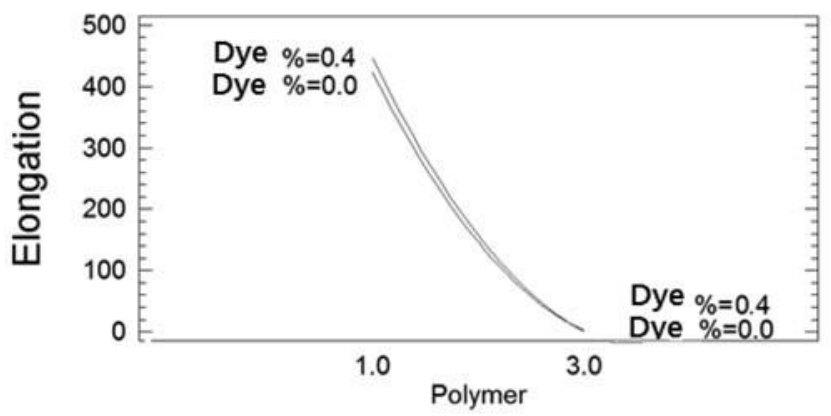

(c)

Interaction Plot for Modulus

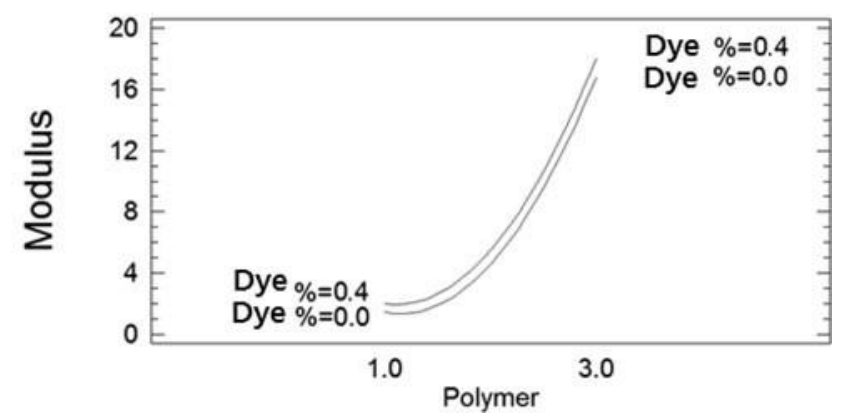

Figure 5. The interaction plots for the tenacity (a), elongation at break (b) and modulus (c).

results are listed in Table 4. The significant factors studied that affect the tenacity are the polymer type $(A)$ and the interaction $A \& A$, the former being greater. There are no significant effects from the other interactions within the factor range used in the set of experiments.

The ANOVA analysis for the elongation shows that polymer type, dye concentration and their interactions ( $A \& A$ and $A \& B$ ) had significant effects on the elongation at break. According to the statistical analyses, the polymer type is the most effective and significant on the modulus. The dye concentration has an effect, but limited and less significant. The effect is dominated by the significance of the polymer type and its interactions.

The geometric result obtained from plotting a response variable as a function of two factors is that the interaction appears as a surface twist (Figure 7). The estimated response surface was based on the regression model as 
discussed in the next section. The estimated response surfaces were used to determine the direction and the significance of the interactions. To determine the direction of the interaction $\mathrm{A} \& \mathrm{~B}$ for each response, the geometric (a) Normal Probability Plot for Tenacity

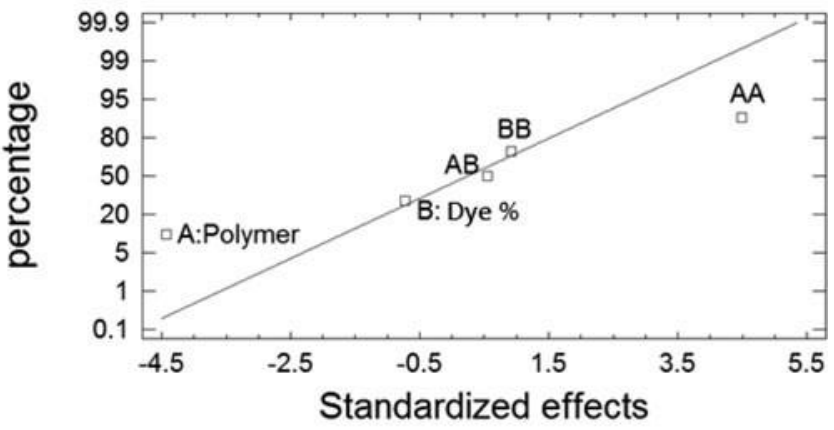

(b) Normal Probability Plot for Elongation

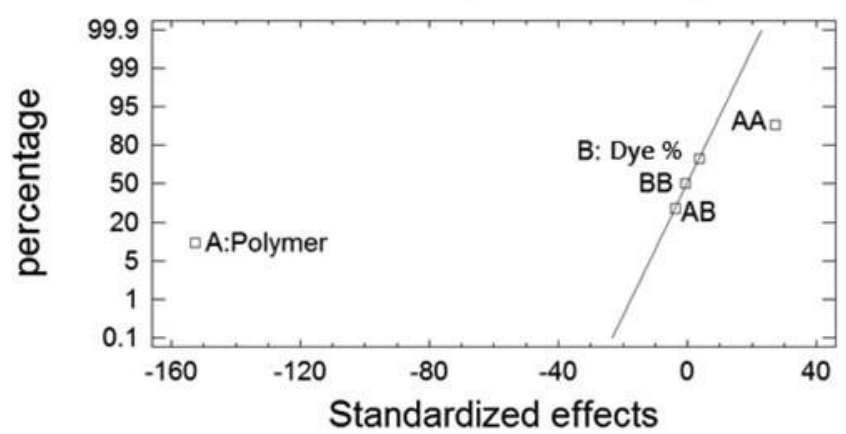

(c) Normal Probability Plot for Modulus

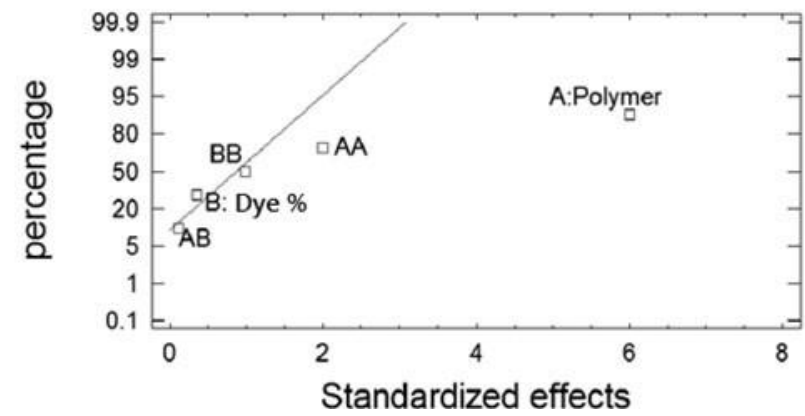

Figure 6. Normal probability plot for the tenacity (a), elongation (b) and modulus (c).

Table 4. Results identifying the statistical significance of factor effects on the results from ANOVA of the data identifying the statistical significance of each factor for tenacity, elongation at break and modulus. p Value

\begin{tabular}{lccc}
\cline { 2 - 4 } Source & Tenacity & Elongation & Modulus \\
\hline A:Polymer & 0.0214 & 0.0000 & 0.0093 \\
B:Dye \% & 0.5207 & 0.0316 & 0.7534 \\
AA & 0.0205 & 0.0001 & 0.1400 \\
AB & 0.6218 & 0.0369 & 0.9199 \\
BB & 0.4265 & 0.5731 & 0.3998 \\
\hline
\end{tabular}

result from plotting the response variable was obtained. There was a notable twist in the elongation plot which confirmed that the interaction was borderline. As there was no twist found in the surface for the tenacity or the modulus, their determined effects were not significant. This observation is consistent with the statistical analysis results presented in the interaction plot and ANOVA derived from the experimental data, as previously discussed.

\begin{tabular}{|c|c|c|c|c|c|c|}
\hline \multirow{2}{*}{ Trial number } & \multirow{2}{*}{ Observed } & \multirow{2}{*}{ Fitted } & \multirow{2}{*}{ Observed } & \multirow{2}{*}{ Fitted } & \\
\hline & & & & & Observed & Fitted \\
\hline 1 & 2.46 & 2.3 & 155.8 & 156.2 & 5.0 & 5.5 \\
\hline 2 & 3.49 & 3.6 & 445.0 & 445.8 & 1.0 & 2.0 \\
\hline 3 & 2.36 & 2.5 & 148.3 & 145.4 & 3.0 & 4.6 \\
\hline 4 & 3.03 & 2.8 & 1.9 & 2.2 & 18.9 & 16.8 \\
\hline 5 & 3.92 & 3.9 & 420.0 & 422.5 & 1.0 & 1.4 \\
\hline 6 & 2.4 & 2.2 & 150.0 & 152.4 & 5.0 & 2.8 \\
\hline 7 & 2.89 & 2.8 & 1.9 & 0.5 & 19.6 & 18.0 \\
\hline 8 & 2.39 & 2.6 & 1.9 & 2.9 & 11.6 & 15.2 \\
\hline 9 & 3.77 & 3.6 & 439.2 & 435.7 & 1.0 & 0.47 \\
\hline
\end{tabular}


(a) Estimated Response Surface

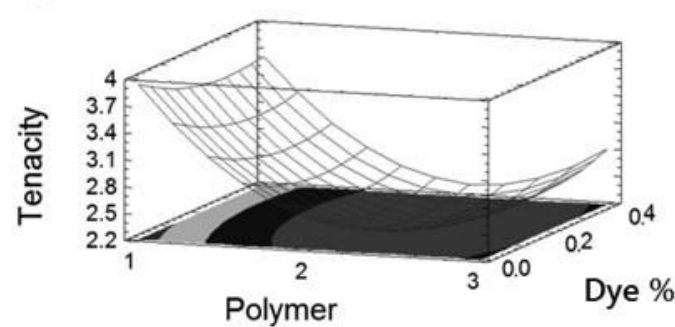

(b)

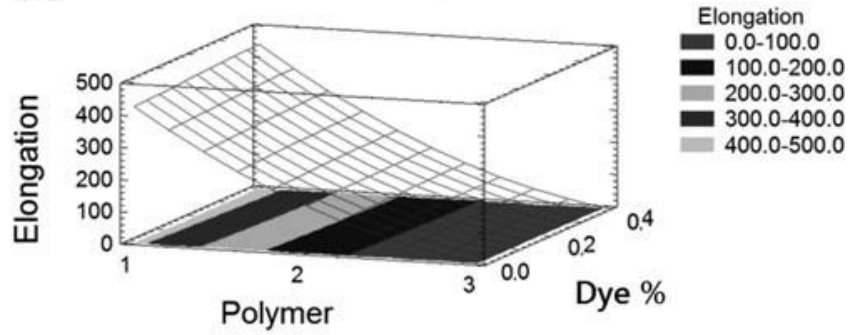

(c)

Estimated Response Surface

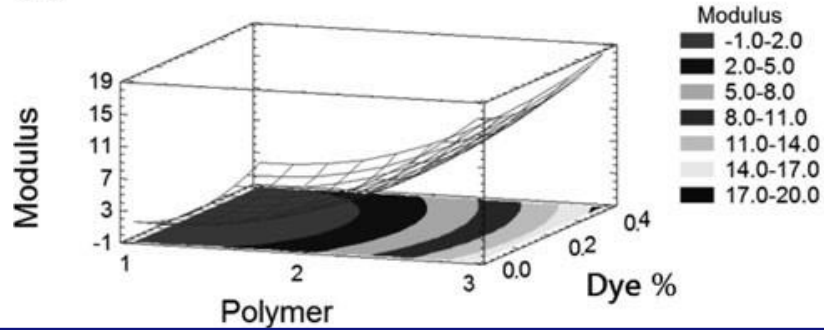

Figure 7. The estimated response surface for the interaction between the factors for the tenacity (a), elongation at break (b) and modulus (c).

Table 5. Estimation results for the tenacity, elongation at break and modulus.

Tenacity value Elongation value Modulus value

\subsubsection{The regression equation and results}

Based on the analysis of the factorial experimental design (L9) results, simplified models based on statistical analysis of the factors studied and their interactions were derived by the regression equations for tenacity, elongation at break and modulus which were obtained by fitting to the experimental data. Enhanced regression equations are useful in forecasting the fibre properties in order to achieve the most satisfactory properties for specific applications. For example, the results of the statistical analysis of a combination of factor levels, as described in this paper, may be used, in principle, to assist the control of photochromic fibre production processes using the regression equations obtained using (a)

Plot of Tenacity

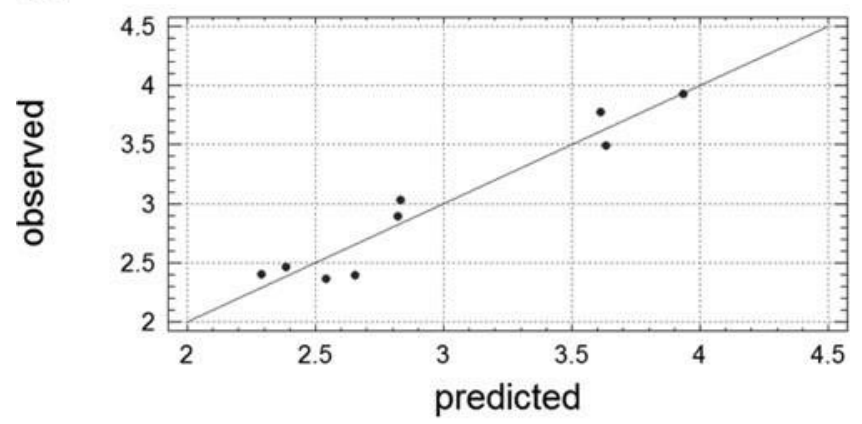

(b)

Plot of Elongation

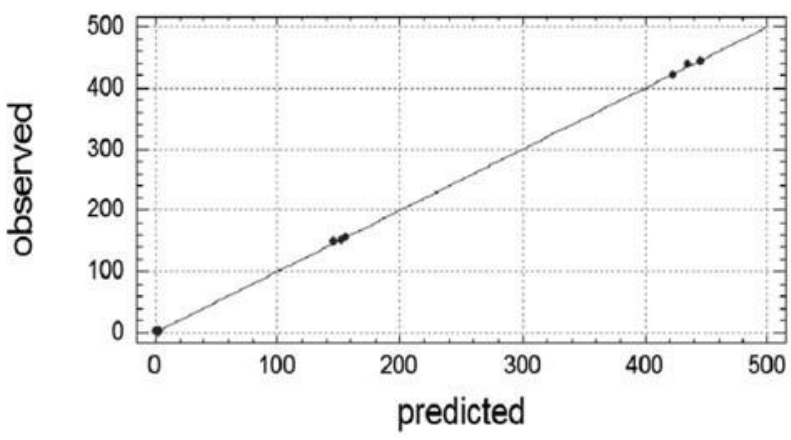

(c)

\section{Plot of Modulus}

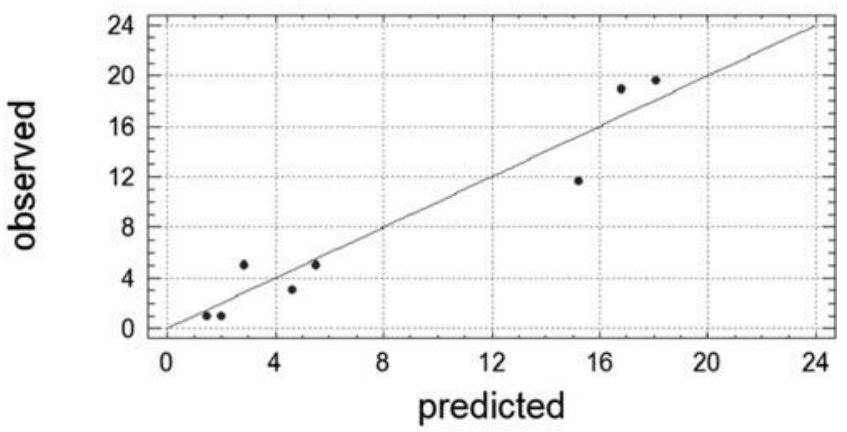

Figure 8. Experimental observed results and calculated fitted results plotted for the tenacity (a), elongation at break (b) and modulus (c). Statgraphics software. The regression equations in terms of the coded values given in Table 1 are as follows:

Tenacity $1 / 4$ 7:01 3:92 A 2:83 B p 0:84 A $^{2}$

$$
\text { p 0:36 A B p 4:29 } B^{2}
$$

Elongation 1/4 833:56 477:96 A p 105: 08 B p 66:95

$$
A^{2} 31: 25 \text { A B }-38: 75 B^{2}
$$

Modulus 1/4 7:33 10:39 A 21:67 B p 4:52 A

$$
\text { p 0:88 A B p 55:42 } \text { B }^{2}
$$


The models evaluate the significance of the effect of each independent variable to a predicted response depending on the coefficient constant for the linear effects of independent factors and the coefficient constant for the regression. Theoretically, the software calculates the standard deviation of the mean values. Then, an estimate is made of the range of values that would encompass the predicted and observed values. The pattern of estimated
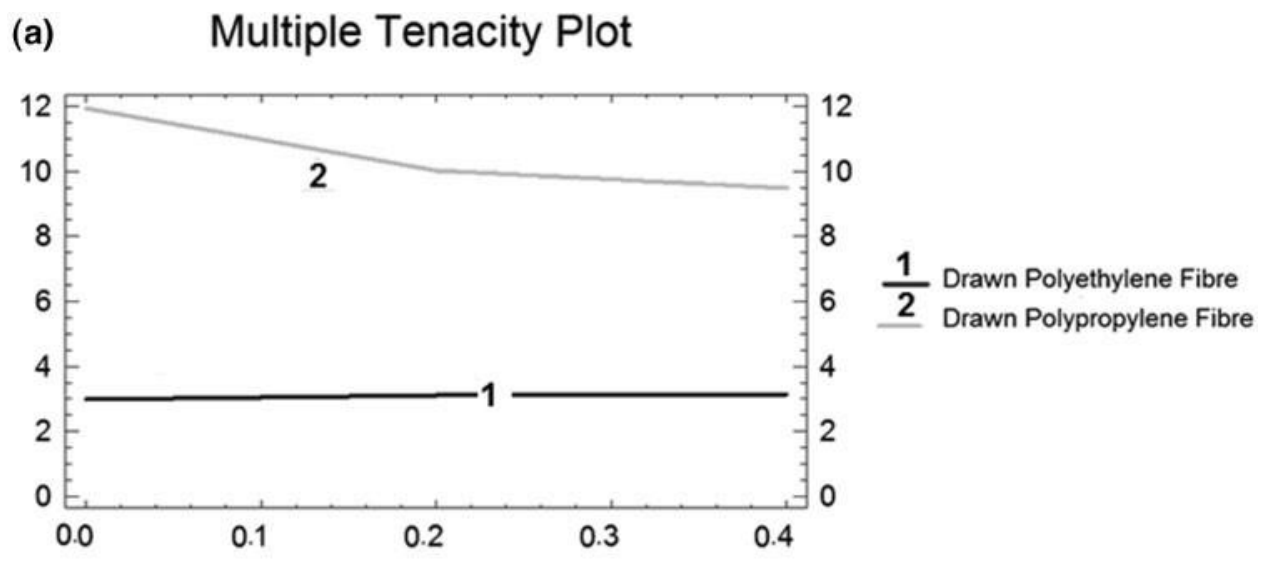

(b) Multiple Elongation Plot

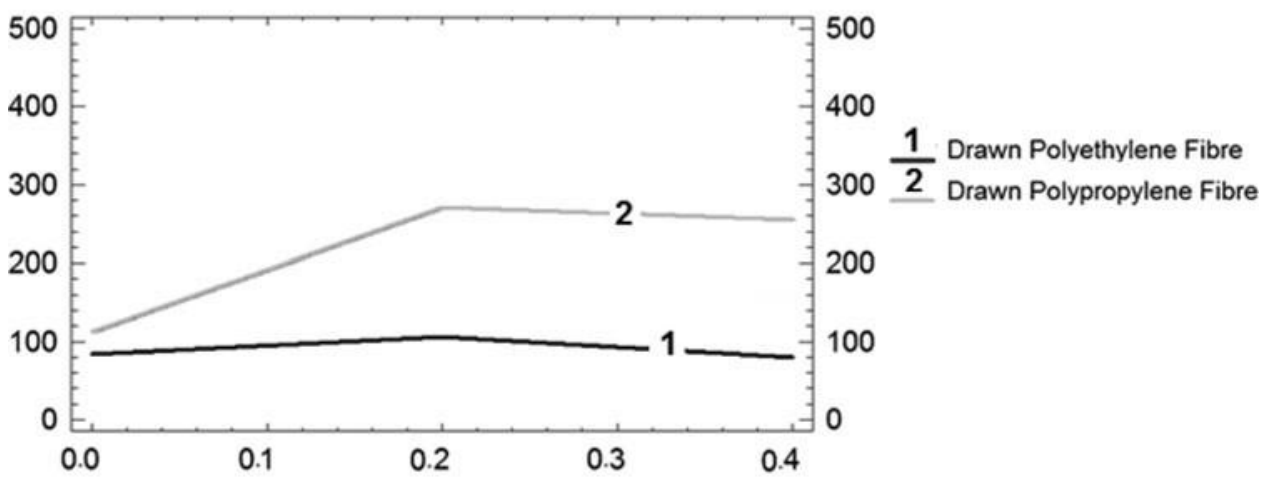

(c) Multiple Modulus Plot

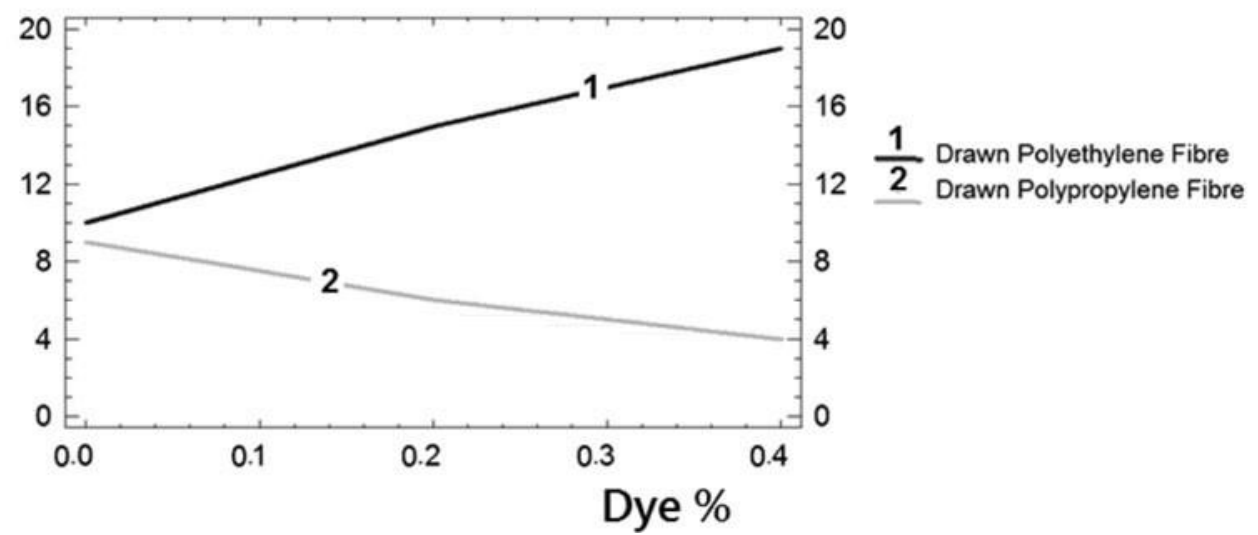

Figure 9. Multiple plot for the drawn fibres of polypropylene and polyethylene: (a) tenacity, (b) elongation, (c) modulus.

interactions effects. Table 5 shows the observed values and the value of each response predicted using the fitted model. Figure 8 shows the observed experimental results and calculated fitted results plotted for (a) tenacity, (b) elongation at break and (c) modulus.

While the point estimate gives the best possible prediction, the prediction cannot be regarded as perfect. The error in prediction could be improved using interval estimates rather than point estimates or by further responses was based on the assumed model derived from the experimental observations.

Thus, it is concluded based on the analyses that the most effective and significant parameter influencing the fibre mechanical properties is the polymer type. Dye concentration has an effect with each of the polymers used but its effect is dominated by the significance of the polymer type and its interactions. 
3.3.2. The effect on the mechanical properties of fibres The mechanical properties of the fibres used in this study are expected, based on literature experience, to depend on the drawing conditions and the original structure of undrawn fibre, which may have para-crystalline or enhancement of the properties. Table 6 also shows the relevant $R^{2}$ and adjusted $R^{2}$ values. The correlation coefficient between the outputs and targets is a measure of how well the variation in the output is explained by the targets, to show the correlation between outputs and

Table 6. The combinations of factor levels for the effect of multi-stage hot drawing on the properties of linear fibres for tenacity, elongation at break and modulus.

\begin{tabular}{|c|c|c|c|c|c|}
\hline \multirow[b]{2}{*}{ Response } & \multirow[b]{2}{*}{$\mathrm{R}^{2}$ (percent) } & \multirow[b]{2}{*}{ Adjusted $\mathrm{R}^{2}$ (percent) } & \multirow[b]{2}{*}{ Optimum model } & \multicolumn{2}{|c|}{$\begin{array}{l}\text { The combination of factor levels } \\
\text { (\#: Low level, -: middle,high level) } \\
\text { ": }\end{array}$} \\
\hline & & & & Polymer & $\begin{array}{l}\text { Dye } \\
\%\end{array}$ \\
\hline Tenacity & 93.2629 & 82.0343 & Maximum & Polyester & $"$ \\
\hline Elongation at break & 99.9875 & 99.9667 & $\begin{array}{l}\text { Minimum } \\
\text { Maximum }\end{array}$ & $\begin{array}{l}\text { Polypropylene } \\
\text { Polypropylene }\end{array}$ & $\begin{array}{l}\# \\
"\end{array}$ \\
\hline Modulus & 93.2012 & 81.8699 & $\begin{array}{l}\text { Minimum } \\
\text { Maximum } \\
\text { Minimum }\end{array}$ & $\begin{array}{l}\text { Polyester } \\
\text { Polypropylene } \\
\text { Polyester }\end{array}$ & "- \\
\hline
\end{tabular}

amorphous character. During hot drawing, molecules are removed from the lamellar crystals, molecules in the amorphous regions are extended, and the mechanical properties are improved. An important issue in this series of investigations is the influence of the dye concentration on the significance of this effect. An ultimate aim would be to optimise the mechanical performance of the photochromic fibres, in relation to the photochromic performance. It is of interest that the dye percentage negatively affects the tenacity of the drawn polypropylene fibres and, in contrast, positively affects the tenacity of the drawn polyethylene fibres, Figure 9(a). A dye percentage of $0.2 \%$ affects positively the elongation of the drawn polypropylene fibres and the drawn polyethylene fibres. The higher dye percentage $(0.4 \%)$ affects negatively the elongation of the drawn polypropylene fibres and the drawn polyethylene fibres, as shown in Figure 9(b). The dye concentration affects negatively the modulus of the drawn polypropylene fibres and positively the modulus of the drawn polyethylene fibres, as shown in Figure 9(c).

According to the analyses, the most effective and significant parameter influencing the fibre mechanical properties is the polymer type. Dye concentration has an effect on each of the polymers used but its effect is dominated by the significance of the polymer type and its interactions.

In this study, the effect of processing on the mechanical properties of the fibres has been characterised and statistically modelled. The statistical models take into account the polymer type and the dye percentage, and their interactions. Table 6 shows the combination of factor levels over the indicated regions which maximise and minimise the responses, namely the tenacity, elongation at break and modulus. The models specified the combinations of the levels that provide an targets, depending on the fitted model generated (Younes, 2012). The coefficient of determination is the ratio of the sum of squares explained by a regression model and the 'total' sum of squares around the mean. A high value does not guarantee that the model fits the data well. To increase the precision of $R^{2}$, an adjusted $R^{2}$ should be used to reflect both the number of independent variables and the sample size in the model; this value will always be less than or equal to $R^{2}$. Using a model that does not fit the data well

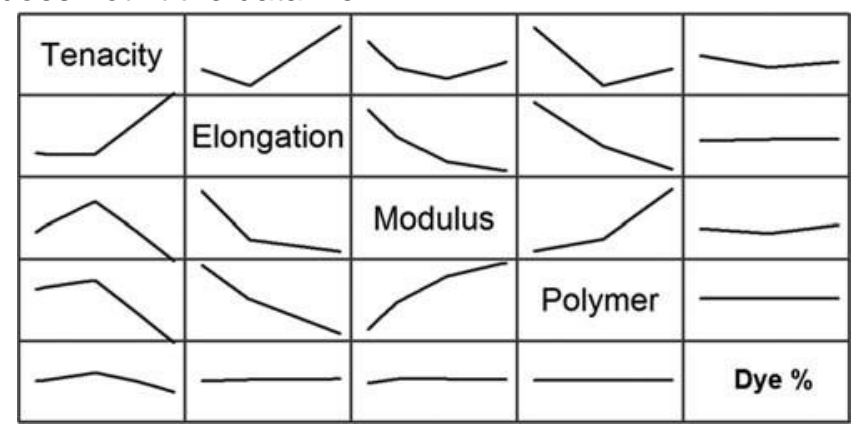

Figure 10. Matrix plot for factors versus tenacity, modulus and elongation at break of fibres.

cannot provide good answers to the underlying engineering or scientific questions under investigation. ANOVA is the proportion of variance which explains the model's terminology and significance. Finally, Figure 10 shows matrix plot for the factors versus tenacity, modulus and elongation at break of fibres and are generally consistent with the previous results.

\section{Conclusion}

As-spun fibres, made from polypropylene and polyethylene, were drawn using similar multi-stage hot- 
drawing process conditions to investigate the effect of incorporating a photochromic dye into the fibres during extrusion. Dye concentration and polymer type affect the tensile properties of the fibres. Mechanical properties are significantly affected by the polymer material used. Other factors and interactions have effects but they are limited and less significant as they are dominated by the governing factor, which is the polymer type. The regression equations obtained form a part of a forecasting program designed to optimise the production process of selected photochromic fibres that will be designed in future. The designed models aim to assist processing scientists and technologists in industry to obtain enhanced properties from the novel fibres under suitable conditions, in relation to final product cost. In addition, new methods and analysis models save time and the cost of production process. It is envisaged that photochromic fibres could be used in chromic textiles for creative design, functional or smart textile applications. Our future work will deal with photochromic behaviour of drawn fibres incorporating a range of dyes in relation to the factors and the interaction effects within the production process.

\section{Acknowledgement}

The authors are indebted to Dr. Alex Fotheringham in the School of Textiles and Design, Heriot Watt University for his greatly valued support. Also thanks go to Dr. Roger Spark in the School of Textiles and Design, Heriot Watt University for his appreciated technical support on testing equipment, and to FMEE, Damascus University for valued support.

\section{Disclosure statement}

No potential conflict of interest was reported by the authors.

\section{References}

Aldib, M., \& Christie, R. M. (2011). Textile applications of photochromic dyes. Part 4: Application of commercial photochromic dyes as disperse dyes to polyester by exhaust dyeing. Coloration Technology, 127, 282-287.

Aldib, M., \& Christie, R. M. (2013). Textile applications of photochromic dyes. Part 5: Application of commercial photochromic dyes to polyester fabric by a solvent-based dyeing method. Coloration Technology, 129(2), 131-143.

Berkovic, G., Krongauz, V., \& Weiss, V. (2000). Spiropyrans and spirooxazines for memories and switches. Chemical Reviews, 100, 1741-1754.

Billah, S. M. R., Christie, R. M., \& Morgan, K. M. (2008). Direct coloration of textiles with photochromic dyes. Part 2: The effect of solvents on the colour change of photochromic textiles. Coloration Technology, 124, 229-233.

Billah, S. M. R., Christie, R. M., \& Shamey, R. (2008). Direct coloration of textiles with photochromic dyes. Part 1: Application of spiroindolinonaphthoxazines as disperse dyes to polyester, nylon and acrylic fabrics. Coloration Technology, 124, 222-223.

Brody, H. (1994). Synthetic fibre materials. London: Longman group UK limited.
Christie, R. M. (2013). Chromic materials for technical textile applications, In M. Gulrajani (Ed.), Advances in the dyeing and finishing of technical textiles. Elsevier.

Christie, R. M., Chi, L. J., Spark, R. A., Morgan, K. M., Boyd, A. S. F., \& Lycka, A. (2005). The application of molecular modelling techniques in the prediction of the photochromic behaviour of spiroindolinonaphthoxazines. Journal of Photochemistry and Photobiology A: Chemistry, 169(1), 37-45.

Corns, S. N., Partington, S. M., \& DTowns, A. (2009). Industrial organic photochromic dyes. Color Technology, 125, 249-261.

Crano, C. J. C., \& Gugliemetti, R. J. (1999). Organic photochromic and thermochromic compounds. New York: Kluwer Academic/Plenum

Publishers.

El-Mogahzy, Y. E. (2009). Engineering textiles: Integrating the design and manufacture of textile products. Cambridge: Woodhead Publishing Limited.

Gardiner, W. P., \& Gettinby, G. (1998). Experimental design techniques in statistical practice, a practical software-based approach.

Chichester: Horwood Publishing Limited.

Hes, L., \& Ursiny, P. (1994). Yarn texturizing technology. Portugal: EEC Comett program \& Eurotex.

Lee, S.-J., Son, Y.-A., Suh, H.-J., Lee, D.-N., \& Kim, S.-H. (2006). Preliminary exhaustion studies of spiroxazine dyes on polyamide fibers and their photochromic properties. Dyes and Pigments, 69(1-2), 18-21.

Little, A. F., \& Christie, R. M. (2010). Textile applications of photochromic dyes. Part 1: Establishment of a methodology for evaluation of photochromic textiles using traditional colour measurement instrumentation. Coloration Technology, 126, 157-163.

Little, A. F., \& Christie, R. M. (2010). Textile applications of photochromic dyes. Part 2: Factors affecting the photocoloration of textiles screen-printed with commercial photochromic dyes. Coloration Technology, 126, 164-170.

Little, A. F., \& Christie, R. M. (2011). Textile applications of photochromic dyes. Part 3: Factors affecting the technical performance of textiles screen-printed with commercial photochromic dyes. Coloration Technology, 127, 275-281.

Little, A. F., \& Christie, R. M. (2016). Textile applications of commercial photochromic dyes. Part 6: Photochromic polypropylene fibres.

Coloration technology, 132, 304-309.

Lochner, R. H., \& Mater, J. E. (1990). Design for quality. London: Chapman and Hall.

Lord, P. (2003). Hand book of yarn production: Technology science and economics. Manchester: The Textile Institute \& CRC \& WP.

Mather, R. R., \& Wardman, R. H. (2011). The chemistry of textile fibres. Royal Society of Chemistry.

Nechwatal, A., \& Nicolai, M. (2011). Interactions between polypropylene and photochromic dyestuffs. Polymer Degradation and Stability, 96, 1648-1652.

Phadke, M. S. (1989). Quality engineering using robust design. New Jersey: Prentice Hall, Englewood Cliffs.

Son, Y.-A., Park, Y.-M., Park, S.-Y., Shin, C.-J., \& Kim, S.-H. (2007). Exhaustion studies of spiroxazine dye having reactive anchor on polyamide fibers and its photochromic properties. Dyes and Pigments, 73(1), 76-80.

Tanguchi, G. (1986). Introduction to quality engineering. Tokyo: Asian productivity organization.

Vlachogiannis, J. G., \& Roy, R. K. (2005). Robust PID controllers by Taguchi method. The TOM Magazine, 17, 456-466. 
Wang, P. Y., \& Wu, C. J. (1997). Photochromic behaviour of some phenoxyanthraquinone dyes in solution and on polyester substrate.

Dyes and Pigments, 35, 279-288.

Younes, B. (2012). The Statistical modelling of production processes of biodegradable aliphatic aromatic co-polyester fibres used in the textile industry (PhD PhD Thesis), Heriot-Watt University, UK. 